\title{
Generalize the Definition of the First Derivative (First-order Principle) to Get a New Definition That Conforms to the Fractional Derivative
}

\author{
Maroti Kamble ${ }^{1}$, Zampalwad S. S \\ Assistant Professor Department of Mathematics, Shri Vitthal Rukhmini Arts Commerce and Science College, Sawanatq. Mahagoan ,dist. \\ Yavatmal Maharashtra, India \\ kamblerajratna2@gmail.com
}

Head Department of Mathematics, Graminmahavidyalaya Vasantnagar Tq. Mukhed Dist.Nanded, Maharashtra, India sambhaji4267@gmail.com

\begin{abstract}
In this paper we define new definition conformable fractional derivative and verify its validity for Linearity property, product rule, Quotient rule, and verify derivative of some Standard function. The definition satisfies the previous results for ordinary derivative and derivative of some standard function. This definition coinside with the classical definition of first order derivative.
\end{abstract}

Keywords: fractional derivative, new definition of conformable fractional derivative

\section{Introduction}

Fractional derivative is as old as calculus. L'Hospital in 1695 asked what does it meanif $\frac{d^{n} f}{d x^{n}}$. If $\mathrm{n}=\frac{1}{2}$ Since then, many researchers tried to put a definition of a fractional derivative. Most of them used an integral form for the fractional derivative.

Two of which are the most popular ones.

1) Riemann liouville definition. For $\alpha \in[n-1, n)$ the $\alpha$ derivative of $\mathrm{f}$ is

$$
D_{a}{ }^{\alpha}=\frac{1}{\Gamma(n-\alpha)} \frac{d^{n}}{d t^{n}} \int_{a}^{t} \frac{f(x)}{(t-x)^{\alpha-n+1}} d x
$$

2) Coputo definition. For $\alpha \in[n-1, n)$ the $\alpha$ derivative of $\mathrm{f}$ is

$$
D_{a}^{\alpha}=\frac{1}{\Gamma(n-\alpha)} \int_{a}^{t} \frac{f^{n}(x)}{(t-x)^{\alpha-n+1}} d x
$$

\section{Methodology}

Now, all definitions including (i) and (ii) above satisfy the property that the fractional derivative is linear. This is the only property satisfied from the first derivative by all of the definitions.

However, the other properties of fractional derivative such as product rule of derivative and derivative of constant is zero and quotient or division rule for derivative, derivative of chain rule following are some of the rules that not satisfied by other definitions:

1) The Riemann-Liouville derivative definition does not satisfy $D_{a}{ }^{\alpha}(1)=0$ butCaputo derivative satisfies $D_{a}^{\alpha}(1)=0$, if $\alpha$ is not a natural number.

2) All fractional derivatives do not satisfy the formula of the derivative of the product of two functions:
3) All fractional derivatives do not satisfy of the formula derivative of the quotient or division of two functions:

4) All fractional derivatives do not satisfy the chain rule:

$$
D_{a}^{\alpha}(f \circ g)=f^{\alpha}(g(t)) g^{\alpha}(t)
$$

5) All fractional derivatives do not satisfy: $D^{\alpha} D^{\beta} f=$ $D^{\alpha+\beta} f$ general.

\section{Models and Analysis}

1) Let $f:[0, \infty) \rightarrow \mathrm{R}$ and $t>0$. definition of the derivative of first principle of fat $t$ is $\frac{d f}{d t}=\lim _{\epsilon \rightarrow 0} \frac{f(t+\varepsilon)-f(t)}{\epsilon}$.

We can give the a similar definition for the fractional derivative of order $\alpha$, where $0<\alpha \leq 1$ and for any general $\alpha$ for $\alpha \in(n, n+1]$ where $n \in \mathrm{N}$.

Definition 3.1. Given a function $f:[0, \infty) \rightarrow \mathrm{R}$. Then the "conformable fractional derivative" of $f$ of order $\alpha \in$ $(0,1]$ is defined by

$T_{\alpha} f(t)=\lim _{\varepsilon \rightarrow 0} \frac{f\left(t_{0}+\varepsilon^{\alpha} t^{1-\alpha}\right)-f\left(t_{0}\right)}{\varepsilon^{\alpha}}$

This definition satisfy all the above five properties of $T_{1}$ of derivative.

For $\alpha=1$

$T_{1} \mathrm{f}(\mathrm{t})=\lim \frac{f\left(t+\varepsilon^{1} t^{1-1}\right)-f(t)}{\varepsilon^{1}}$

$=\lim _{\epsilon \rightarrow 0} \frac{f(t+\varepsilon)-f(t)}{\epsilon}$

Reduces to classical definition of derivative

Let us write $T_{\alpha}$ to denote the operator which is called the fractional derivative of order $\alpha$. For $\alpha=1, T 1$ satisfies the following properties:

(i) $T_{1}(a f \pm b g)=a T_{1}(g) \pm b T_{1}(f)$, for all $a, b \in \mathrm{R}$ and $f, g$ in the domain of $T_{1}$.

(ii) $T_{1}\left(t^{p}\right)=p t^{p-1}$ 
(iii) $T_{1}(f g)=f T_{1}(g)+g T_{1}(f)$

(iv) $T_{1}\left(\frac{f}{g}\right)=\frac{g T_{1}(f)-f T_{1}(g)}{g^{2}}$

(v) $T_{1}(\lambda)=0$, for all constant functions $f(t)=\lambda$.

2) Now, we present our new definition, which is the simplest and most natural extension of derivative definition of first principle. and efficient definition of fractionalderivative of order $\alpha \in(0,1]$.the definition can be generalized to include any $\alpha$.

\section{Results and Discussion}

For all $t>0, \alpha \in(0,1)$. If $f$ is $\alpha$-differentiable in some $(0, a)$, $a>0$, and $\lim _{t \rightarrow 0^{+}} f^{\alpha}(t)$ exists then define $\lim _{t \rightarrow 0^{+}} f^{\alpha}(t)=$ $f \propto 0$

$f^{\alpha}(t), T_{\alpha} f(t)$ are same notation for the conformable fractional derivatives of $f$ of order $\alpha$., $f$ is $\alpha$-differentiable.if the new conformable fractional derivative of $f$ of order $\alpha$ exists from the definition above following theorem can obtain.

Theorem 3.1. If a function $f:[0, \infty)-\rightarrow \mathrm{R}$ is $\alpha$-differentiable at $t_{0}>0$,

$\alpha \in(0,1]$, then $f$ is continuous at $t_{0}$.

\section{Proof:}

Since $f\left(t_{0}+\varepsilon^{\alpha} t^{1-\alpha}\right)-f\left(t_{0}\right)=\frac{f\left(t_{0}+\varepsilon^{\alpha} t^{1-\alpha}\right)-f\left(t_{0}\right)}{\varepsilon^{\alpha}} \cdot \varepsilon^{\alpha}$ then

$$
\begin{gathered}
\lim _{\varepsilon \rightarrow 0} f\left(t_{0}+\varepsilon^{\alpha} t^{1-\alpha}\right)-f\left(t_{0}\right)=\lim _{\varepsilon \rightarrow 0} \frac{f\left(t_{0}+\varepsilon^{\alpha} t^{\varepsilon^{\alpha}-\alpha}\right)-f\left(t_{0}\right)}{\varepsilon^{\alpha}} \cdot \lim _{\varepsilon \rightarrow 0} \varepsilon^{\alpha} \\
\lim _{\varepsilon \rightarrow 0} f\left(t_{0}+\varepsilon^{\alpha} t^{1-\alpha}\right)-f\left(t_{0}\right)=f^{\alpha}\left(t_{0}\right) .0 \\
\lim _{\varepsilon \rightarrow 0} f\left(t_{0}+\varepsilon^{\alpha} t^{1-\alpha}\right)-f\left(t_{0}\right)=0
\end{gathered}
$$

Which implies that

Hence $f$ is continuous.

$$
\lim _{\varepsilon \rightarrow 0} f\left(t_{0}+\varepsilon^{\alpha} t^{1-\alpha}\right)=f\left(t_{0}\right)
$$

Theorem 3.2: Let $\alpha \in(0,1]$ and $f, g$ be $\alpha$-differentiable at a point $t>0$. Then

(i) $T_{\alpha}(a f+b g)=a T_{\alpha}(f)+b T_{\alpha}(g)$, for all $a, b \in \mathrm{R}$ and

$f, g$ in the domain of $T_{1}$.

i) $T_{\alpha}(a f+b g)=$

$$
\begin{aligned}
& \lim _{\varepsilon \rightarrow 0} \frac{a f\left(t_{0}+\varepsilon^{\alpha} t^{1-\alpha}\right)+b g\left(t_{0}+\varepsilon^{\alpha} t^{1-\alpha}\right)-\left\{a f\left(t_{0}\right)+b g\left(t_{0}\right)\right\}}{\varepsilon^{\alpha}} \\
&= \lim _{\varepsilon \rightarrow 0} \frac{a f\left(t_{0}+\varepsilon^{\alpha} t^{1-\alpha}\right)-a f\left(t_{0}\right)+\left\{b g\left(t_{0}+\varepsilon^{\alpha} t^{1-\alpha}\right)-b g\left(t_{0}\right)\right\}}{\varepsilon^{\alpha}} \\
&= \lim _{\varepsilon \rightarrow 0} \frac{a\left\{f\left(t_{0}+\varepsilon^{\alpha} t^{1-\alpha}\right)-f\left(t_{0}\right)\right\}+b\left[g\left(t_{0}+\varepsilon^{\alpha} t^{1-\alpha}\right)-g\left(t_{0}\right)\right]}{\varepsilon^{\alpha}} \\
&= a \lim _{\varepsilon \rightarrow 0} \frac{f\left(t_{0}+\varepsilon^{\alpha} t^{1-\alpha}\right)-f\left(t_{0}\right)}{\varepsilon^{\alpha}} \\
&+b \cdot \lim _{\varepsilon \rightarrow 0} \frac{g\left(t_{0}+\varepsilon^{\alpha} t^{1-\alpha}\right)-g\left(t_{0}\right)}{\varepsilon^{\alpha}}
\end{aligned}
$$

(ii) $T_{\alpha}(a f-b g)=a T_{\alpha}(g)-b T_{\alpha}(f)$, for all $a, b \in \mathrm{R}$ and $f, g$ in the domain

of $T_{1}$.

$$
\begin{aligned}
& T_{\alpha}(a f-b g)= \\
& \lim _{\varepsilon \rightarrow 0} \frac{a f\left(t_{0}+\varepsilon^{\alpha} t^{1-\alpha}\right)-b g\left(t_{0}+\varepsilon^{\alpha} t^{1-\alpha}\right)-\left\{a f\left(t_{0}\right)-b g\left(t_{0}\right)\right\}}{\varepsilon^{\alpha}} \\
&= \lim _{\varepsilon \rightarrow 0} \frac{a f\left(t_{0}+\varepsilon^{\alpha} t^{1-\alpha}\right)-a f\left(t_{0}\right)-\left\{b g\left(t_{0}+\varepsilon^{\alpha} t^{1-\alpha}\right)-b g\left(t_{0}\right)\right\}}{\varepsilon^{\alpha}} \\
&= \lim _{\varepsilon \rightarrow 0} \frac{a\left\{f\left(t_{0}+\varepsilon^{\alpha} t^{1-\alpha}\right)-f\left(t_{0}\right)\right\}-\left\{b\left[g\left(t_{0}+\varepsilon^{\alpha} t^{1-\alpha}\right)-g\left(t_{0}\right)\right]\right\}}{\varepsilon^{\alpha}} \\
&= a \lim _{\varepsilon \rightarrow 0} \frac{f\left(t_{0}+\varepsilon^{\alpha} t^{1-\alpha}\right)-f\left(t_{0}\right)}{\varepsilon^{\alpha}} \\
&-b \cdot \lim _{\varepsilon \rightarrow 0} \frac{g\left(t_{0}+\varepsilon^{\alpha} t^{1-\alpha}\right)-g\left(t_{0}\right)}{\varepsilon^{\alpha}}
\end{aligned}
$$

(iii) $T_{\alpha}\left(t^{p}\right)=p t^{p-\alpha}$ for all $\mathrm{p} \in R$ this is same as for first order derivative.

$$
\begin{aligned}
& \begin{array}{c}
T_{\alpha}\left(t^{p}\right)=\lim _{\varepsilon \rightarrow 0} \frac{\left(t+\varepsilon^{\alpha} t^{1-\alpha}\right)^{p}-t^{p}}{\varepsilon^{1-\alpha}} \\
=\lim _{\varepsilon \rightarrow 0} \frac{t^{p}+p t^{p-1} \varepsilon^{\alpha} t^{1-\alpha}+\frac{P(P-1)}{2} t^{p-2} \varepsilon^{\alpha^{2}} t^{(1-\alpha)^{2}}+\cdots \ldots \ldots \ldots \ldots \ldots \ldots \ldots+t^{p}}{\varepsilon^{\alpha}} \\
=\lim _{\varepsilon \rightarrow 0} \frac{p t^{p-1} \varepsilon^{\alpha} t^{1-\alpha}+\frac{P(P-1)}{2} t^{p-2} \varepsilon^{\alpha^{2}} t^{(1-\alpha)^{2}}+\cdots \ldots \ldots \ldots}{\varepsilon^{\alpha}}
\end{array} \\
& =\lim _{\varepsilon \rightarrow 0} p t^{p-1} t^{1-\alpha}+\frac{\frac{P(P-1)}{2} t^{p-2} \varepsilon^{\alpha^{2}} t^{(1-\alpha)^{2}}+\cdots \cdots \cdots \cdots}{\varepsilon^{\alpha}} \\
& =p t^{p-\alpha} \text {. }
\end{aligned}
$$

(iv) $T_{\alpha}(f g)=f T_{\alpha}(g)+g T_{\alpha}(f)$ i.e derivative of product is same as product of derivative for new conformable fractional derivative.

$$
\text { i) } \begin{aligned}
T_{\alpha}(a f+b g)=\lim _{\varepsilon \rightarrow 0} \frac{a f\left(t_{0}+\varepsilon^{\alpha} t^{1-\alpha}\right)+b g\left(t_{0}+\varepsilon^{\alpha} t^{1-\alpha}\right)-\left\{a f\left(t_{0}\right)+b g\left(t_{0}\right)\right\}}{\varepsilon^{\alpha}} \\
=\lim _{\varepsilon \rightarrow 0} \frac{a f\left(t_{0}+\varepsilon^{\alpha} t^{1-\alpha}\right)-a f\left(t_{0}\right)-\left\{b g\left(t_{0}+\varepsilon^{\alpha} t^{1-\alpha}\right)-b g\left(t_{0}\right)\right\}}{\varepsilon^{\alpha}} \\
=\lim _{\varepsilon \rightarrow 0} \frac{a\left\{f\left(t_{0}+\varepsilon^{\alpha} t^{1-\alpha}\right)-f\left(t_{0}\right)\right\}+b\left[g\left(t_{0}+\varepsilon^{\alpha} t^{1-\alpha}\right)-g\left(t_{0}\right)\right]}{\varepsilon^{\alpha}} \\
=a \lim _{\varepsilon \rightarrow 0} \frac{f\left(t_{0}+\varepsilon^{\alpha} t^{1-\alpha}\right)-f\left(t_{0}\right)}{\varepsilon^{\alpha}}+b \cdot \lim _{\varepsilon \rightarrow 0} \frac{g\left(t_{0}+\varepsilon^{\alpha} t^{1-\alpha}\right)-g\left(t_{0}\right)}{\varepsilon^{\alpha}} \\
=a T_{\alpha} f+b . T_{\alpha} g .
\end{aligned}
$$


(v)

$T_{\alpha}(f / g)=\frac{g T_{\alpha}(f)-f T_{\alpha}(g)}{g^{2}}$ this can follow from definition.

(vi) $T_{\alpha}(\lambda)=0$, for all constant functions $f(t)=\lambda$. i.e. derivative for constant is zero.

vii) If in addition, $\mathrm{f}$ is differentiable then $T_{\alpha}(f)(t)=$ $t^{1-\alpha} \frac{d}{d t} f(t)$

$T_{\alpha}(f)(t)=t^{1-\alpha} \frac{d}{d t} f(t)$

$\lim _{\varepsilon \rightarrow 0} \frac{f\left(t+\varepsilon^{\alpha} t^{1-\alpha}\right)-f(t)}{\varepsilon^{\alpha}}$
Let $=h=\varepsilon^{\alpha} t^{1-\alpha}=\varepsilon^{\alpha} t^{1-\alpha} \quad$ implies $\quad \varepsilon^{\alpha}=h t^{\alpha-1} \quad$ as $\varepsilon \rightarrow$ $0, h \rightarrow 0$

$$
\begin{aligned}
& =\lim _{h \rightarrow 0} \frac{f(t+h)-f(t)}{h t^{\alpha-1}} \\
& =\lim _{h \rightarrow 0} \frac{f(t+h)-f(t)}{h t^{\alpha-1}} \\
& =t^{1-\alpha} \frac{d}{d t} f(t) .
\end{aligned}
$$

Fractional derivatives of certain functions from new definition for comformable fractional derivative.

$$
\begin{gathered}
1 . T_{\alpha}\left(t^{p}\right)=p t^{p-\alpha} \text { for all } p \in R \\
T_{\alpha}\left(t^{p}\right)=\lim _{\varepsilon \rightarrow 0} \frac{\left(t+\varepsilon^{\alpha} t^{1-\alpha}\right)^{p}-t^{p}}{\varepsilon^{1-\alpha}} \\
=\lim _{\varepsilon \rightarrow 0} \frac{t^{p}+\quad p t^{p-1} \varepsilon^{\alpha} t^{1-\alpha}+\frac{P(P-1)}{2} t^{p-2} \varepsilon^{\alpha^{2}} t^{(1-\alpha)^{2}}+\ldots \ldots \ldots \ldots \ldots \ldots \ldots+t^{p}}{p t^{p-1} \varepsilon^{\alpha} t^{1-\alpha}+\frac{P(P-1)}{2} t^{\alpha-2} \varepsilon^{\alpha^{2}} t^{(1-\alpha)^{2}}+\cdots \ldots \ldots \ldots} \\
\varepsilon^{\alpha} \\
=\lim _{\varepsilon \rightarrow 0} p t^{p-1} t^{1-\alpha}+\frac{\frac{P(P-1)}{2} t^{p-2} \varepsilon^{\alpha^{2}} t^{(1-\alpha)^{2}}+\cdots \ldots \ldots \ldots}{\varepsilon^{\alpha}} \\
=p t^{p-\alpha} .
\end{gathered}
$$

2. $T_{\alpha}(1)=0$ follows from 1 by putting $1=t^{0}$

3. $T_{\alpha}\left(e^{c x}\right)=c x^{1-\alpha} e^{c x}, c \in R$

$T_{\alpha}\left(e^{c x}\right)=\lim _{\varepsilon \rightarrow 0} \frac{e^{\left(c x+c \varepsilon^{\alpha} t^{1-\alpha}\right)-e^{c t}}}{\varepsilon^{\alpha}}$

$T_{\alpha}\left(e^{c x}\right)=\lim _{\varepsilon \rightarrow 0} \frac{e^{c t} e^{c \varepsilon^{\alpha} t^{1-\alpha}}-e^{c t}}{\varepsilon^{1-\alpha}}$

Here $h=h=\varepsilon^{\alpha} t^{1-\alpha} \Rightarrow \varepsilon^{\alpha}=h . t^{\alpha-1}$

Putting the values

$$
\begin{aligned}
& T_{\alpha}\left(e^{c t}\right)=\lim _{\varepsilon \rightarrow 0} \frac{e^{c t}\left(e^{c h}-1\right)}{h \cdot t^{\alpha-1}} \\
& T_{\alpha}\left(e^{c x}\right)=\lim _{h \rightarrow 0} \frac{e^{c t}\left(e^{c h}-1\right) \cdot c}{c \cdot h \cdot t^{\alpha-1}} \\
& T_{\alpha}\left(e^{c x}\right)=\frac{e^{c t} c}{t^{\alpha-1}} \lim _{h \rightarrow 0} \frac{\left(e^{c h}-1\right) .}{c \cdot h .} \\
& \text { as } \varepsilon \rightarrow 0, \Rightarrow h \rightarrow 0 \\
& T_{\alpha}\left(e^{c t}\right)=\frac{e^{c t} c}{t^{\alpha-1}} \cdot 1 \\
& T_{\alpha}\left(e^{c t}\right)=c t^{1-\alpha} e^{c t}, c \in R . \\
& \text { 4) } T_{\alpha}(\operatorname{sinbx})=\text { bx } x^{1-\alpha} \operatorname{cosbx}, b \in R \\
& \text { By using definition. }
\end{aligned}
$$$$
=\lim _{\varepsilon \rightarrow 0} \frac{\sin b\left(x+\varepsilon^{\alpha} x^{1-\alpha}\right)-\sin b x}{\varepsilon^{\alpha}}
$$$$
=\lim _{\varepsilon \rightarrow 0} \frac{2 \cos b\left(x+\frac{\varepsilon^{\alpha} x^{1-\alpha}}{2}\right) \sin \frac{b \varepsilon^{\alpha} x^{1-\alpha}}{2}}{\varepsilon^{1-\alpha}}
$$

$$
\begin{aligned}
& =\cos b x \lim _{\varepsilon \rightarrow 0} \frac{\sin \frac{b \varepsilon^{\alpha} x^{1-\alpha}}{2}}{\frac{b \varepsilon^{\alpha} x^{1-\alpha}}{2}} \cdot b x^{1-\alpha} \\
& =\cos b x \cdot b x^{1-\alpha}
\end{aligned}
$$

In similar mannar as above $T_{\alpha}(\cos b x)=-b x^{1-\alpha} \sin b x, b \in$ $R$ can be proved.

However, it is worth noting the following conformable fractional derivatives of certain functions:
4. $T_{\alpha}\left(e^{\frac{1}{\alpha} t^{\alpha}}\right)=\left(e^{\frac{1}{\alpha} t^{\alpha}}\right)$
5. $T_{\alpha}\left(\sin \frac{1}{\alpha} t^{\alpha}\right)=\left(\sin \frac{1}{\alpha} t^{\alpha}\right)$
6. $T_{\alpha}\left(\cos \frac{1}{\alpha} t^{\alpha}\right)=\left(\cos \frac{1}{\alpha} t^{\alpha}\right)$

Although the most important case for the range $\alpha$ is $(0,1)$ but if $\alpha \in(n, n+1]$ for some natural number $\mathrm{n}$ then the definition is given by following.

Definition 3.2 Let if $\alpha \in(n, n+1]$, and $\mathrm{f}$ be an $\mathrm{n}$ differentible at $\mathrm{t}$,where $t>0$ then the new definition of comformable fractional derivative of $\mathrm{f}$ of order $\alpha$ is defined as

$$
\begin{aligned}
& T_{\alpha} f(t) \\
& =\lim _{\varepsilon \rightarrow 0} \frac{f^{(\lceil\alpha]-1)}\left(t+\varepsilon^{\lceil\alpha\rceil} t^{(\lceil\alpha\rceil-\alpha)}-f^{(\lceil\alpha]-1)}(t)\right.}{\varepsilon^{\lceil\alpha\rceil}}
\end{aligned}
$$

Where $\lceil\alpha\rceil$ is the smallest integer greater than or equal to $\alpha$.

Remark 3.1: one can easily prove that

$$
T_{\alpha} f(t)=t^{([\alpha]-\alpha)} f^{\lceil\alpha]}(t)
$$

Where $\alpha \in(n, n+1]$ and $\mathrm{f}$ is $\mathrm{n}+1$ differentiable at $t>0$ 
$T_{\alpha} f(t)=\lim _{\varepsilon \rightarrow 0} \frac{f^{([\alpha]-1)}\left(t+\varepsilon^{\lceil\alpha]} t^{([\alpha]-\alpha)}-f^{([\alpha]-1)}(t)\right.}{\varepsilon^{[\alpha]}}$

Put $h=\varepsilon^{[\alpha]} t^{([\alpha]-\alpha)}$ then $\varepsilon^{[\alpha]}=h . t^{(\alpha-[\alpha])}$ as $\varepsilon \rightarrow 0$ then $h \rightarrow$ 0

$$
\begin{gathered}
T_{\alpha} f(t)=\lim _{\varepsilon \rightarrow 0} \frac{f^{(\lceil\alpha]-1)}(t+h)-f^{(\lceil\alpha]-1)}(t)}{h \cdot t^{(\alpha-\lceil\alpha])}} \\
T_{\alpha} f(t)=t^{([\alpha]-\alpha)} \lim _{\varepsilon \rightarrow 0} \frac{f^{(\lceil\alpha]-1)}(t+h)-f^{(\lceil\alpha]-1)}(t)}{h} \\
T_{\alpha} f(t)=t^{(\lceil\alpha\rceil-\alpha)} f^{\lceil\alpha\rceil}(t)
\end{gathered}
$$

\section{Conclusion}

We introduced new definition of conformable fractional derivative.

We conclude that this definition coinside with the classical definition of derivative.

We introduce definition For $\alpha \in(0,1]$ and generalize for any $\in(n, n+1]$.

\section{References}

[1] K.S. Miller, An Introduction to Fractional Calculus and Fractional Differential Equations, J. Wiley and Sons, New York, 1993.

[2] K. Oldham, J. Spanier, The Fractional Calculus, Theory and Applications of Differentiation and Integration of Arbitrary Order, Academic Press, USA, 1974.

[3] A. Kilbas, H. Srivastava, J. Trujillo, Theory and Applications of Fractional Differential Equations, in: Math. Studies., North-Holland, New York, 2006.

[4] I. Podlubny, Fractional Differential Equations, Academic Press, USA, 1999.

[5] R. khalil, M. AlHorani, A. Yousef, M. Sababheh " A new Defination of Fractional derivative", journel of computational and applied mathematics . 